\title{
Nordamerikanische Pflanzen im Palmengarten
}

\section{Hilke STEINECKE}

\section{Verschiedene Aktivitäten}

Der Palmengarten beherbergt in seinem Freiland eine große Zahl nordamerikanischer Parkbäume, darunter auch Amberbaum und ZuckerAhorn. In der nordamerikanischen Prärie blühen im Sommer viele prächtige Stauden wie Purpur-Sonnenhut und Goldrute. Zudem sind im Sommer-Sukkulentengarten zahlreiche Kakteen, Palmlilien und Agaven zu bestaunen. Viele dieser Gewächse sind traditionelle Nahrungs-, Heil- oder Zeremonialpflanzen, die seit Jahrhunderten von den Ureinwohnern Amerikas genutzt werden. Bei dieser großen Auswahl nordamerikanischer Pflanzen, zu denen es viele spannende Geschichten gibt, bietet es sich an, Führungen und Aktionen zu „Indianerpflanzen“ anzubieten. Auf dem Programm der Grünen Schule stehen zum Thema "Indianerpflanzen“ Kindergeburtstage sowie 1,5-stündige Führungen. In abendlicher Atmosphäre wurde das Thema „Indianerpflanzen“ auch im Sommer 2007 während zweistündiger Führungen umgesetzt, die hier vorgestellt werden sollen.

\section{Indianer - die Ureinwohner Amerikas}

Unter Indianern versteht man die Volksgruppen, die Amerika schon vor der europäischen Kolonialisierung bewohnt haben. Sie werden auch als indigene Völker Amerikas bezeichnet. Manche verstehen unter Indianern nur die Ureinwohner Nordamerikas, während mit der spanischen Bezeichnung Indios die vorkolumbianischen $\mathrm{Be}$ wohner Mittel- und Südamerikas gemeint sind. Die Ureinwohner Nordamerikas waren und sind kein einheitliches Volk, auch wenn sie häufig unter einem Begriff zusammengefasst werden. Es gab viele verschiedene Völker, von den Apachen bis hin zu den Zuni. Ebenso vielfältig waren ihre Sprachen. Ganz grob und vereinfacht werden die Indianer in die sogenannten Küsten-, PuebloPrärie- und Wald-Indianer eingeteilt. Zu Beginn der Führung wurde ein Überblick über ihre Lebensgewohnheiten, Ernährung und Siedlungstypen gegeben.
Die Ureinwohner Amerikas sind sehr naturverbunden und achten Tiere, Pflanzen und Steine, mit denen sie sich auch schmückten. Für die Bewohner Mexikos war Türkis ein heiliger Stein, mit dem Zeremonienmasken verziert wurden. Noch heute ist der Navajo-Schmuck, Silberschmiede-Arbeiten in Kombination mit Türkis, von Neu-Mexiko bis nach Kalifornien sehr beliebt und eine wichtige Einnahmequelle für die indianische Bevölkerung. An der ersten Pflanzen-Station durften die aromatischen Blätter des Amberbaumes (Liquidambar styraciflua) beschnuppert werden. Der Harzbalsam spielte beispielsweise in der Zahnpflege eine Rolle. Nicotiana sylvestris im Sommerflor war Anlass, die Bedeutung von Tabak sowohl als rituelles Heilmittel als auch als „Füllmaterial“ für die Friedenspfeife zu erklären.

\section{Nordamerikanische Waldbäume}

Im Spätsommer und Herbst färben sich an der Ostküste Nordamerikas, von Nord nach Süd fortschreitend, die Wälder leuchtend gelb bis rot. Man spricht von „Indian Summer“. Nach indianischer Mythologie fließt das Blut der im Herbst erlegten Bären in die Erde, wird von den Bäumen aufgenommen und manifestiert sich in der Blattfarbe. Eine besonders auffällige Herbstfärbung zeigen Amberbaum sowie verschiedene Ahorn-, Walnuss-, Hickory- und Eichen-Arten. Diese waren auch die nächsten Stationen.

An der Schwarznuss (Juglans nigra) waren bereits die dicken grünen Früchte zu sehen. Ihre gerbstoffhaltigen Blätter und die Rinde wurden gegen Entzündungen eingesetzt. Schwarznuss war, wie bei uns die Walnuss, eine wichtige Färbepflanze. Stoffe und Wolle lassen sich mit dem Sud aus Blättern oder grünen Fruchtschalen goldbraun färben. Eng verwandt mit den Wal- sind die Hickorynüsse, deren bekanntester Vertreter die Pekannuss (Carya illinoinensis) ist. Nur wenige Schritte weiter gab es an einem Ahorn den berühmten Kanadischen Zucker-Ahorn (Acer saccharum) auf einer Waffel zu kosten. Spannend war 
auch die Erzählung, wie die Menschen überhaupt darauf gekommen sind, den Blutungssaft des Zucker-Ahorns anzuzapfen. Eicheln und Rosskastanien gibt es auch bei uns zu Genüge, jedoch wird sie außer in Notzeiten wohl kaum jemand sammeln, um aus ihnen Mehl herzustellen, da ihnen zunächst die Bitter- und Gerbstoffe entzogen werden müssen. Die Indianer legten Kastanien (z. B. von Aesculus pavia) zusammen mit heißen Steinen in Erdlöcher, worin sie langsam gegart wurden. Anschließend wurden sie geschält und zerkleinert. Der Brei wurde in einem Sieb vier Tage lang gewässert, somit entbittert, und dann an der Sonne getrocknet. Aus dem Mehl buken sie Brote und Pfannkuchen.

\section{Im trockenen Süden}

Im Sommer-Sukkulentengarten gab es einiges über Pflanzen aus den Trockengebieten der südwestlichen USA zu erfahren. Aus getrockneten und entkernten Yuccafrüchten bereiteten die Pueblo-Indianer Süßigkeiten. In diesem Zusammenhang durften Erläuterungen zur Symbiose zwischen Yuccamotte und Yucca nicht fehlen.

Indianer hatten viele Symbole, die der Natur entlehnt waren. Häufig zu Schmuck verarbeitet und deshalb auch bei uns bekannt ist der Flötenspieler (Kokopelli). Nach verschiedenen Legenden bringt er den Menschen Mais oder einen Sack voller Lieder. Er gilt auch als Regenmacher, der mit seinen Melodien die Regenwolken anlockt. Hier war dann auch leicht der Bezug zum Mais herzustellen. Mais war eine der wichtigsten Nahrungspflanzen der Indianer. Meist wurde Mais zusammen mit Bohnen und Kürbissen in Mischkultur angebaut. Die Geschichte von der alten Maismutter, die den Menschen den Mais brachte, erklärte, warum aus den Maiskolben fädige Strukturen heraushängen: Es sind nicht die eingetrockneten Griffel, vielmehr die Haare der Maismutter. Am Seerosenbecken konnte zudem ein weiteres wichtiges Nutzgras, der Indianerreis (Zizania aquatica), betrachtet werden.

Abb. 1 (oben): Amberbaum mit beginnender Herbstfärbung. Abb. 2 (Mitte): Schwarznüsse in ihrer grünen Hülle.

Abb. 3 (unten): Kanadische Goldrute, bei uns ein häufiger Neophyt.
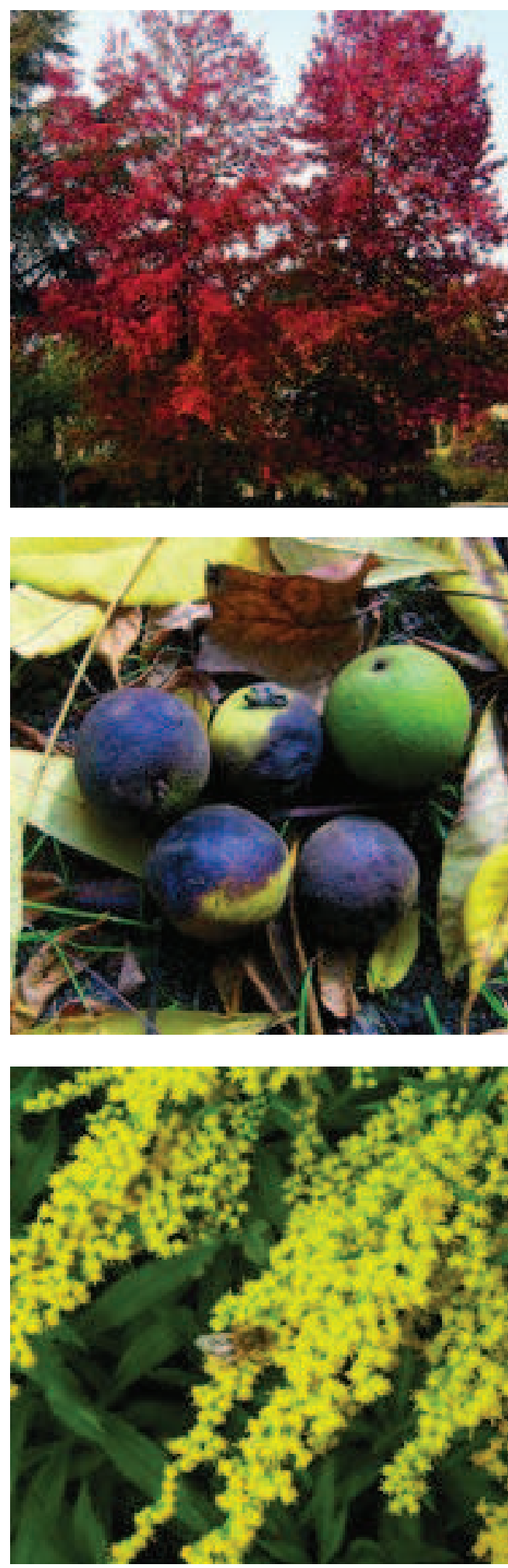


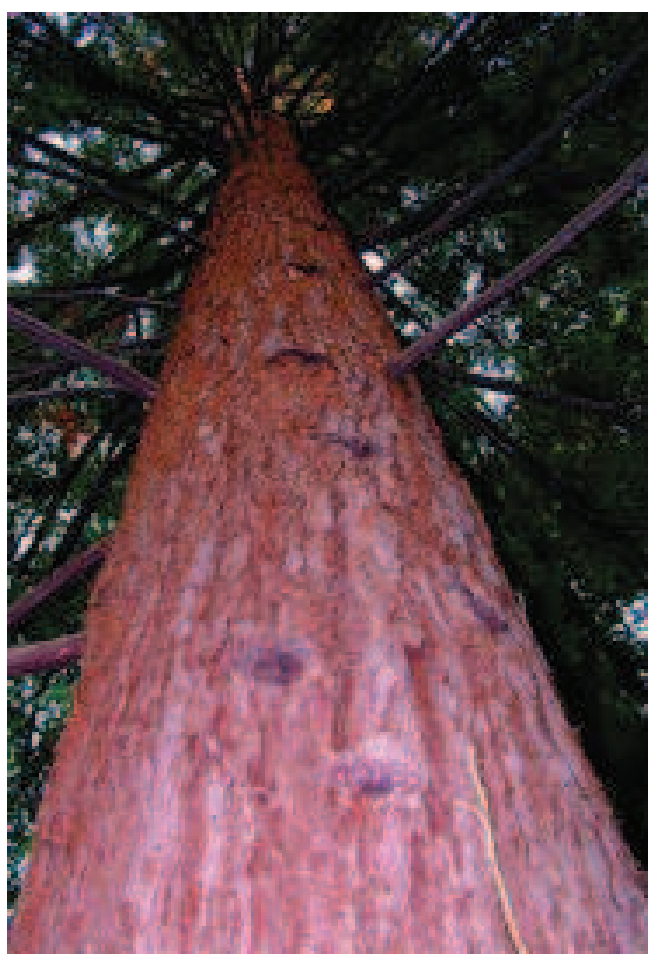

\section{In der Prärie}

Weiter ging es entlang der Steppenanlage bis zur nordamerikanischen Prärie. Dort blühten die auch bei uns als Heilpflanze bekannte Echinacea purpurea und die zum Färben verwendete Goldrute (Solidago speciosa). Zu diesen beiden Arten sowie zur Sonnenblume (Helianthus anuus) gab es viel Wissenswertes über ihre Nutzungen zu erfahren. Mit der Sonnenblume eng verwandt ist Topinambur (Helianthus tuberosus), Hauptnahrungsmittel mancher Indianer. Der Name geht auf einige Vertreter des Volksstammes Tupinambou zurück. Einige dieser Menschen wurden als „Geschenk" nach Frankreich mitgebracht. Dieser Begriff wurde für Bizarres, Fremdes und Neues verwendet, so auch für die bereits im 17 . Jh. in Frankreich angebaute Topinambur-Pflanze. Für kultische Räucherungen wurden Präriesalbei (Salvia azurea) und Beifuß-Arten, ebenfalls Salbei genannt, verwendet. Die Führungsteilnehmer konnten sich von dem starken aromatischen Duft eines Räucherzopfes überzeugen.

In der Prärie warteten ein Tipi sowie Berichte über die Büffeljagd. Ein recht schweres Anschau- ungsobjekt war dabei ein original eiszeitlicher Wisentschädel aus dem Mainzer Becken. Europäische Wisente sind sehr eng mit den amerikanischen Bisons (auch Indianerbüffel genannt) verwandt. Bevor die Weißen die Bisons extrem reduzierten, wurden sie in Maßen von den Indianern gejagt. Bisonfleisch war Grundlage für Pemmikan, eine nahrhafte und haltbare Mischung aus zerstoßenem Dörrfleisch und Fett, oft in Kombination mit Beeren, die als Reiseproviant und Notration verwendet wurde.

Für viele war es neu, dass William F. CODY, besser bekannt als BUFFALO BILL, seine erfolgreiche Wildwest-Show um 1890 auch im Palmengarten aufgeführt hat. Seine Show war äußerst beliebt, in München kamen 18 Tage lang jeweils 8000 Zuschauer.

\section{Mammutbäume}

Die Gruppe versammelte sich gegen Ende der Führung im Sequoia-Hain. Der Redwood oder Küsten-Mammutbaum wurde nach SEQUOYAH (1770-1843), Sohn einer Cherokee-Indianerin und eines europäischen Händlers, benannt. Er entwickelte eine Laut-Silbenschrift für die Cherokee-Sprache. Die 86 Zeichen repräsentieren sämtliche in dieser Sprache vorkommenden Silben. Die letzte Station befand sich am großen Weiher neben dem Riesen-Mammutbaum (Sequioadendron giganteum). Mit Blick auf den nächtlichen Sternenhimmel klang die Führung mit einem indianischen Märchen über die Bärenjagd aus. Die Bären entschwanden in den Himmel und erscheinen heute im Sternbild des Großen Wagens oder des Großen Bären.

Weitere Anregungen zur Gestaltung von Indianerpflanzen-Führungen sind der Handreichung des Verbandes Botanischer Gärten (WÖHRMANN 2003) zu entnehmen.

\section{Literatur}

WÖHRMANN, F. 2003: Die Pflanzenwelt der Indianer.

Indianerpflanzen in Botanischen Gärten. - Ergebnisse der 7. Fortbildung der AG Pädagogik im Verband Botanischer Gärten e. V. - ergänzt durch Anregungen und Handreichungen für die praktische Arbeit. - Frankfurt. (Zu beziehen über den Palmengarten Frankfurt)

Abb. 4: Der rötliche, bereits recht dicke Stamm des RiesenMammutbaumes am Großen Weiher. 